\title{
Chemical Analysis with Single Atom Sensitivity Using Aberration-Corrected STEM
}

Robert F. Klie ${ }^{1}$, Ahmet Gulec, ${ }^{1}$ JingJing Liu, ${ }^{2}$ Tadas Paulauskas, ${ }^{1}$ Patrick J. Phillips, ${ }^{1}$ Canhui Wang ${ }^{1}$ and Randall J. Meyer ${ }^{2}$

1. Department of Physics, University of Illinois at Chicago, Chicago, IL 60607, USA

2. Department of Chemical Engineering, University of Illinois at Chicago, Chicago, IL 60607, USA

The last few years have seen a paradigm change in scanning transmission electron microscopy, STEM, with unprecedented improvements in both spatial and spectroscopic resolution being realized by aberration correctors, cold-field emission guns and monochromators. The successful correction of lens aberrations has greatly advanced the ability of the STEM to provide direct, real space imaging at atomic resolution. Very complementary to reciprocal space methods, this is especially advantageous for aperiodic systems, nanostructures, interfaces and point defects. Aberration-correction has also enabled the development of new imaging techniques, such as incoherent annular bright field (ABF) imaging, which enables the direct visualization of light atoms, such as hydrogen or lithium. While these instrumentation developments have brought notable successes in materials analysis, in particular for hetero-interfaces, catalysis and thin-film studies, they have also challenged the established experimental protocols and our fundamental understanding of both imaging and spectroscopy in the STEM. Aberration correction also allows increased flexibility in choosing the appropriate electron energy to minimize beam induced damage while maintaining atomic-resolution (e.g. $60 \mathrm{keV}$ electrons for studying graphene with $1.3 \AA$ resolution).[1]

In this presentation, we will present the latest results from the new probe aberration-corrected cold-field emission JEOL JEM-ARM200CF at the University of Illinois at Chicago (UIC), which allows in-situ characterization with $78 \mathrm{pm}$ spatial resolution and an energy resolution of $350 \mathrm{meV}$ in the temperature range between $10 \mathrm{~K}$ and $1,300 \mathrm{~K}$ using a variety of in-situ heating, cooling, tomography and electrical feedback holders. The primary electron energy can be chosen between 80 and $200 \mathrm{kV}$. We will show how low-energy imaging can now be used to characterize beam-sensitive materials without significant loss in spatial resolution and how such experiments enable direct correlation with other techniques, including atom-probe tomography and first-principles modeling.

Figure 1 shows an atomic-resolution high-angle annular dark-field image (HAADF) of edges in single layer $\mathrm{MoS}_{2}$ with a primary beam energy of $80 \mathrm{kV}$. While even at $80 \mathrm{kV}$ electron energy-loss spectroscopy of core-loss edges, such as the sulphur K-edge, is still impossible, the images have been used to determine the edge termination of MoS2 sheets and explain their exceptionally high $\mathrm{CO}_{2}$ reduction performance.[3]

Using the newly installed Oxford Instruments X-Max 100TLE, a $100 \mathrm{~mm}^{2}$ silicon drift detector on our JEOL ARM200CF, we can acquire atomic-resolution STEM-XEDS maps in as short as 1 minute for elements as light as Al. Figure 2 shows such an atomic resolution STEM-XEDS map of CdTe (110) after a $\mathrm{CdCl}_{2}$ annealing step. As can be seen in the spectrum image, both the $\mathrm{Cd}$ and Te atomic columns are resolved in addition to the $\mathrm{Cl}$ signal at the center of a dislocation core. Spectrum images like this can be used to determine the effects of $\mathrm{Cl}$ segregation in CdTe solar-cell devices and sees the structural changes as the results of the $\mathrm{CdCl}_{2}$ post-growth annealing step. [4] 


\section{References:}

[1] Zhou, W., J. Lee, J. Nanda, S T. Pantelides, S.J. Pennycook, and J.-C. Idrobo, Atomically localized plasmon enhancement in monolayer graphene. Nature Nanotechnology, 2012. 7(3): p. 161-165. [2] R. F. Klie, A. Gulec, Z. Guo, T. Paulauskas, Q. Qiao, R. Tao, C. Wang, K. B. Low, A. W. Nicholls, and P. J. Phillips, Crystal Research and Technology, 1-12 (2013)

[3] Mohammad Asadi, Bijandra Kumar, Amirhossein Behranginia, Brian A. Rosen, Artem Baskin, Nikita Repnin, Davide Pisasale, Patrick Phillips, Wei Zhu, Richard Haasch, Robert F. Klie, Petr Král, Jeremiah Abiade, Amin Salehi-Khojin, (submitted)

[4] This work is supported by the US Department of Energy (DE-EE-0005956) and the National Science Foundation (DMR-0959470, DMR-0846748, CBET-1067020)

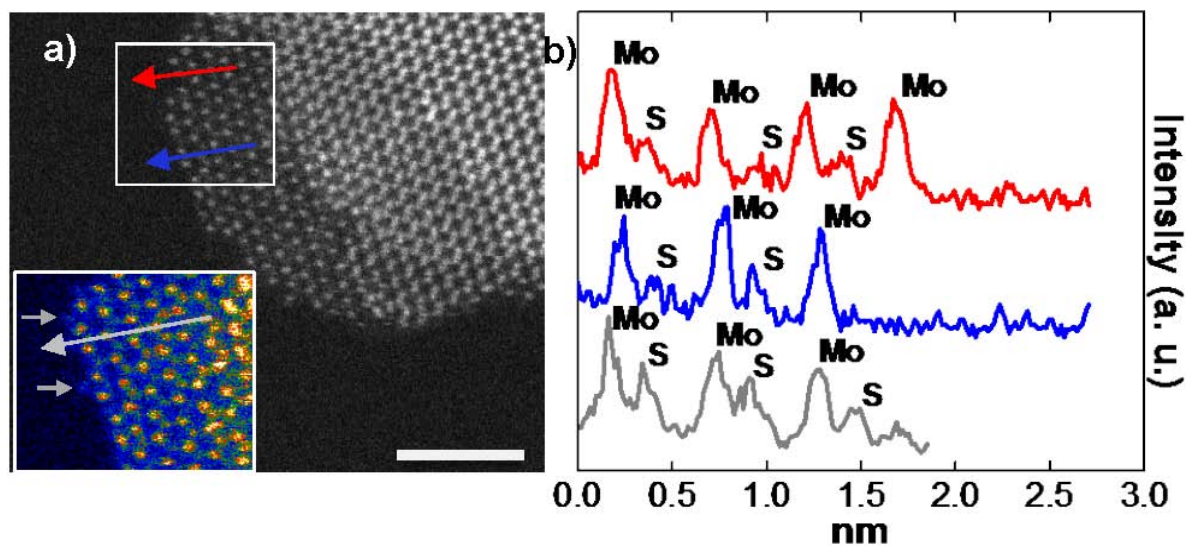

Figure 1. a) Raw grayscale HAADF and false-color low-angle annular dark-field (LAADF) image (inset) of MoS2 edges (scale bar $-5 \mathrm{~nm}$ ) and $\mathrm{b}$ ) the line scans (red and blue towards edges) identifying Mo atoms to be the terminating atoms in the general case. In limited instances, an additional light atom (gray line scan) occupying what should be a Mo-position, most probably a carbon atom from the STEM substrate.
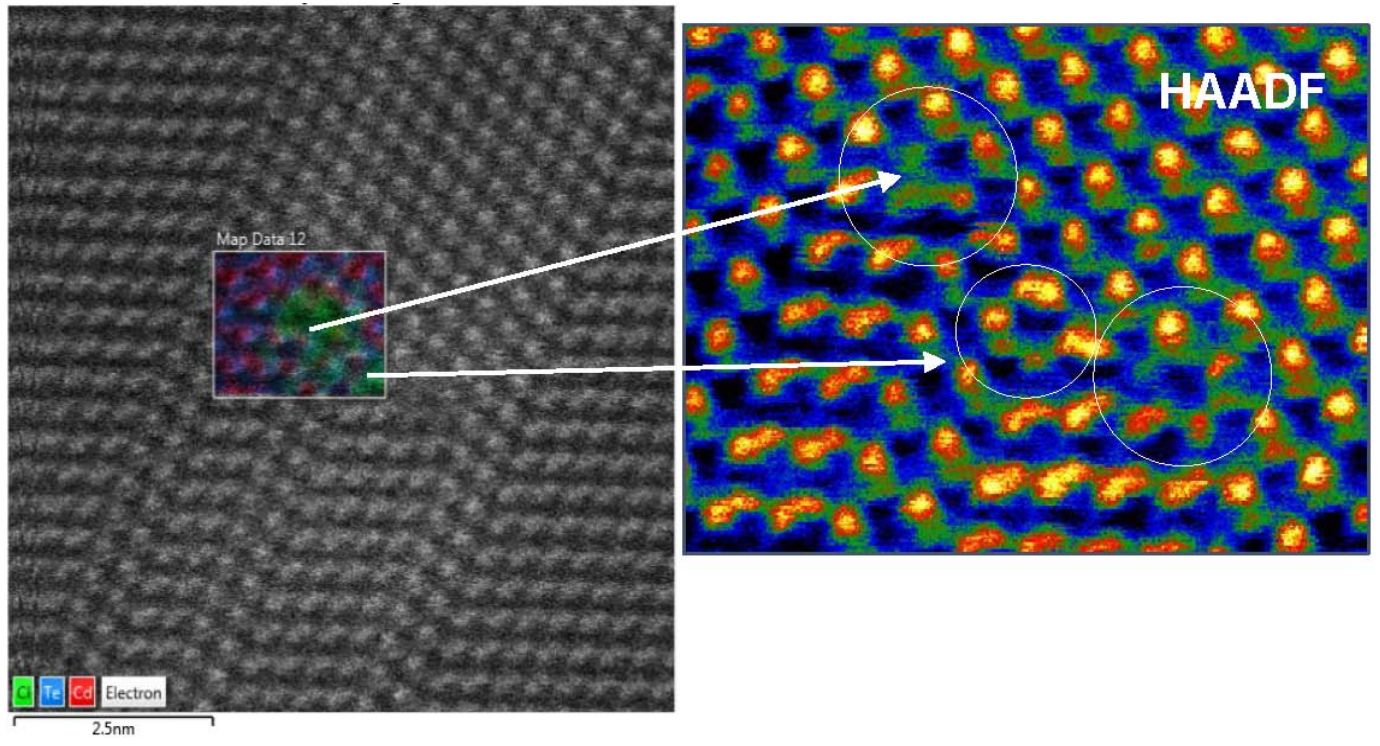

Figure 2. Atomic-resolution XEDS map complex dislocation core in CdTe 